\title{
miR-455-5p promotes cell growth and invasion by targeting SOCO3 in non-small cell lung cancer
}

\author{
Junfeng Wang ${ }^{1}$, Yanbo Wang ${ }^{1}$, Dawei Sun ${ }^{1}$, Jianlong Bu${ }^{1}$, Fenghai Ren ${ }^{1}$, Benkun \\ Liu ${ }^{1}$, Shibo Zhang ${ }^{1}$, Zigeng $X \mathbf{u}^{1}$, Sainan Pang ${ }^{1}$ and Shidong $\mathbf{X u}^{1}$ \\ ${ }^{1}$ The Department of Thoracic Surgery, Harbin Medical University Cancer Hospital, Harbin 150081, China \\ Correspondence to: Shidong XU, email: xusd163@163.com \\ Keywords: miR-455-5p; SOCO3; invasion; non-small cell lung cancers \\ Received: January 23, $2017 \quad$ Accepted: August 29, $2017 \quad$ Published: November 20, 2017 \\ Copyright: Wang et al. This is an open-access article distributed under the terms of the Creative Commons Attribution License 3.0 \\ (CC BY 3.0), which permits unrestricted use, distribution, and reproduction in any medium, provided the original author and source \\ are credited.
}

\section{ABSTRACT}

Non-small cell lung cancer (NSCLC) is the most common type of lung cancer. miR-455-5p has increased expression and the ability to promote tumorigenesis in certain cancers. However, the role of miR-455-5p in NSCLC has not been sufficiently investigated. SOCS3 (suppressor of cytokine signaling 3 ), an important tumor suppressor, is often aberrantly inactivated in various tumors, but it is currently unclear whether SOCO3 is a target of miR-455-5p. In the present study, we investigated the role of miR-455-5p in NSCLC. We found that the expression of miR-455-5p was upregulated in NSCLC tumor tissues compared to corresponding noncancerous tissues, and its expression was correlated with metastasis and tumor node metastasis in NSCLC tissue. We then showed that miR-455-5p promoted migration, invasion and proliferation in NSCLC cell lines. Additionally, we also found that SOCS3 was the direct target gene of miR-455-5p. Consistently, the expression of SOCS3 was negatively correlated with the expression of miR-455-5p in NSCLC tissues. We further show that aberrant miR-455-5p expression is partially controlled by activated ERK signaling in NSCLC. Therefore, miR-455-5p could enhance the growth and metastasis of NSCLC by inhibiting SOCS3, thus providing a potential molecular therapeutic target for the treatment of NSCLC patients.

\section{INTRODUCTION}

Lung cancer becomes a main reason of cancer mortality throughout the world $[1,2]$. Non-small cell lung cancer (NSCLC) is a major type cancer. NSCLC with metastasis is the primary cause of lung cancer mortality [3-5]. Thus, exploring the mechanisms of NSCLC tumorigenesis could be very helpful for NSCLC treatment through identifying effective therapies.

It is well-known that microRNAs (miRNAs) do not encode protein sequences, but negatively control mRNA stability and/or inhibit mRNA translation [6-8]. They can play important roles in various stages of tumorigenesis and tumor development [9]. Depending on the different target genes, miRNAs can promote or inhibit tumorigenesis [10, 11]. Accumulating reports indicated miR-455-5p is critical for many cancers. For example, miR-455-5p expression levels correlated with clinicopathological features and thus served as a prognostic and diagnostic biomarker in endometrial serous adenocarcinomas [12], basal cell carcinoma [13], laryngeal cancer [14], and hepatocellular adenoma [15]. It has been reported that miR-455-5p promoted melanoma metastasis by inhibiting CPEB1 [16]. However, the function of miR-455-5p in NSCLC is currently unknown.

In this study, we explored the function of miR-455$5 \mathrm{p}$ in NSCLC development. Our results showed that miR455-5p promotes tumor growth and metastasis through inhibiting SOCS3 in NSCLC. 


\section{RESULTS}

\section{miR-455-5p is up-regulated in human NSCLC samples and correlated to NSCLC progression}

To find out the function of miR-455-5p in NSCLC cells, we firstly detected miR-455-5p expressionin 79 pairs of human NSCLC tissues and their comparable nontumor tissue. miR-455-5p expression was notably up-regulated in tumor tissues $(58 \%)$ compared with the controls (Figure 1A and 1B). The levels of miR-455-5p were increased in advanced lung cancer (stage III, $n=46$ ) to beginning stages (stage I, $n=$ 33) (Figure 1C). Moreover, miR-455-5p expression was upregulated in metastatic NSCLC $(n=50)$ compared to NSCLC that non-spread $(n=29)$ (Figure 1D).

\section{SOCS3 is a target of miR-455-5p}

Using the computational prediction program TargetScan, we found that suppressor of cytokine signaling (SOCS) 3, which plays a role in the feedback inhibition of JAK/STAT signaling [17-19], was a supposed target of miR-455-5p (Figure 2A). To explore whether miR455-5pregulates SOCS3, the wild-type SOCS3 3'-UTR was cloned, and transfected the reporters together with control RNA or miR-455-5p mimics. Co-transfection of miR-455-5p mimics notably blocked ( 1.7 fold) the wildtype reporter activity, in contrast, the mutant reporter was almost not affected (Figure 2B), suggesting that SOCS3 is a target of miR-455-5p.

Then, we compared miR-455-5p expression in a normal lung cell line and NSCLC cell lines. qRT-PCR was employed to detect its expression in several cell lines, including H460, SK-MES-1, SPCA-1, A549, and H1299, and the normal lung cell line 16HBE. miR-455-5p was highly expressed in all NSCLC cell lines examined. In contrast, its expression was low in the normal lung cell line 16HBE (Supplementary Figure 1). We transfected miR-455-5pmimics in $\mathrm{H} 460$ cells, a decreased endogenous level of miR-455-5p, and tested the endogenous SOCS3 expression by Western blot assay. The level of SOCS3 protein was inhibited ( 2 fold) in cells with miR-455-

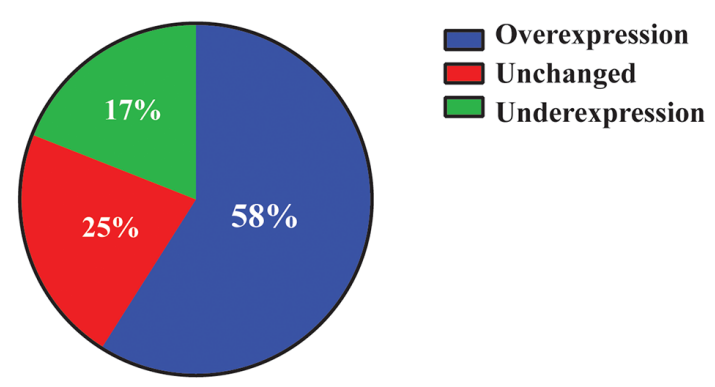

C

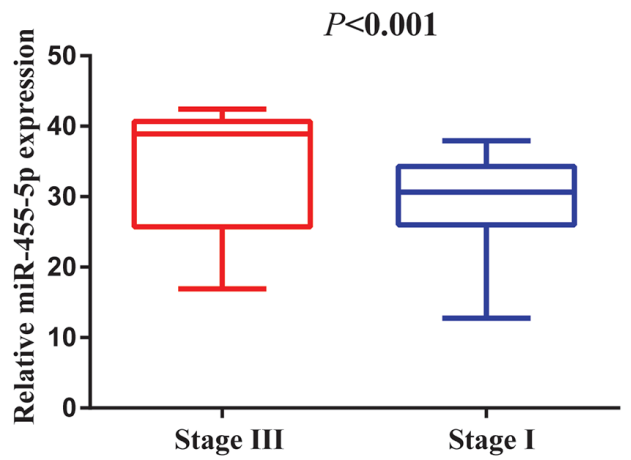

B

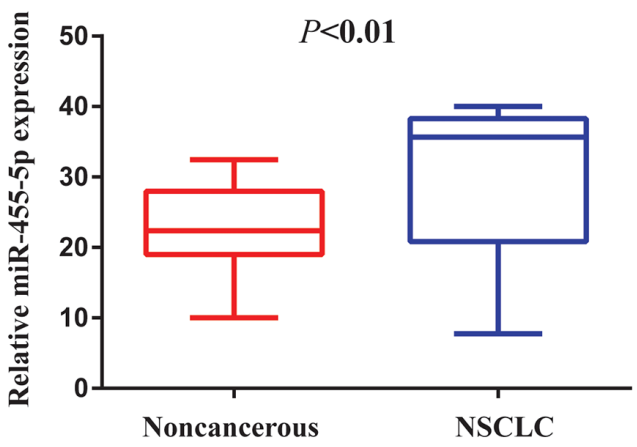

D

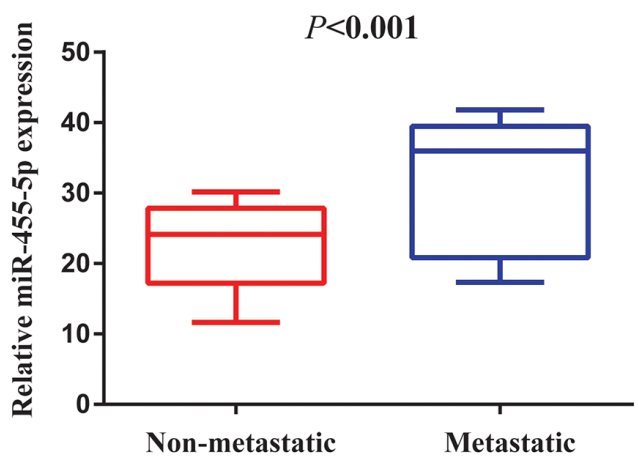

Figure 1: miR-455-5p is up-regulated in human NSCLC tissues and associated with NSCLC progression. (A) The expression levels of miR-455-5p in 79 paired NSCLC and corresponding noncancerous tissues were measured by TaqMan real-time PCR and normalized against an endogenous U6 RNA control. (B) The expression of miR-455-5p was overexpressed in NSCLC tissues compared with the noncancerous tissues. (C) miR-455-5p expression was detected in different clinical stages of NSCLC. (D) The up-regulation of miR-455$5 p$ in NSCLC was associated with tumor metastasis; the patients were classified into tumor metastasis negative and positive groups (lymph nodemetastasis and/ or distal metastasis) Error bars represent SEM. The qRT-PCR were analyzed and shown as relative miR-455-5p levels of the $\mathrm{Ct}$ (cycle threshold) values. The statistical analysis was performed using paired $t$ test (B) and Student's $t$ test (C) and (D). 
A

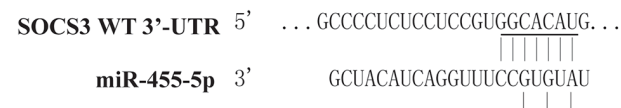

SOCS3 Mutant 3'-UTR 5’ ... GCCCCUCUCCUCCGUCCCGCGUG. .

C
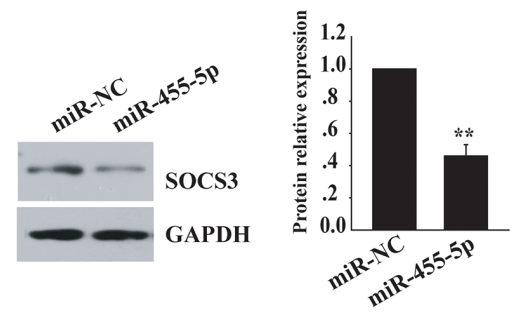

B

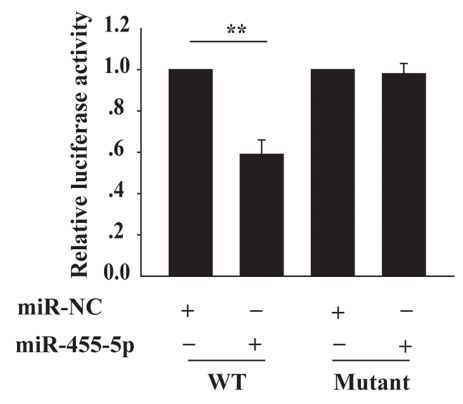

D

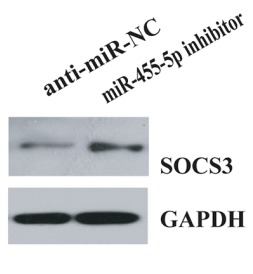

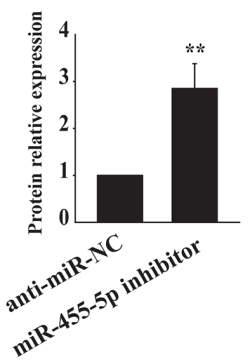

Figure 2: SOCS3 is a target of miR-455-5p. (A) SOCS3is predicted to be a target of miR-455-5p. The sequences of the putative miR455-5p binding sites in wild type and mutant (red) SOCS3-3'UTR. (B) SOCS3 3'-UTR luciferase reporter assays in 293T cells. (C) Western blot analysis of SOCS3 protein levels in miR-455-5p mimics transfected H460 cells. (D) Western blot analysis of SOCS3 protein levels in antimiR-455-5p-transfected H1299 cells. Error bars represent SD. ${ }^{* *}, \mathrm{P}<0.01$. Results shown are representative of three independent experiments.

A

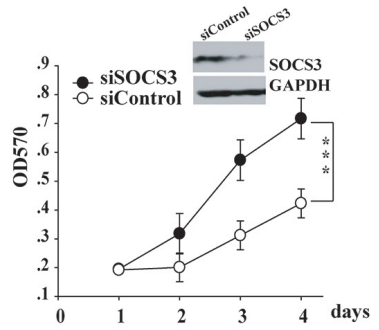

D

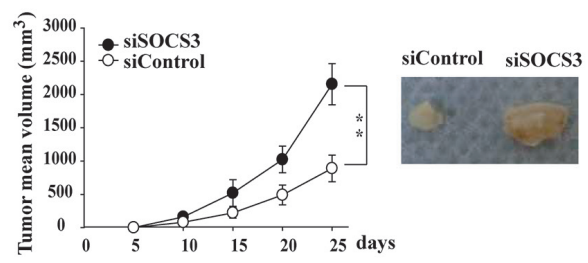

B

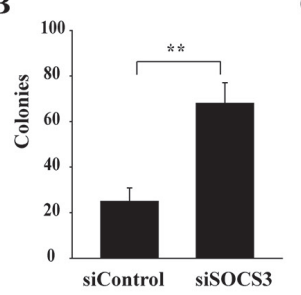

E
C

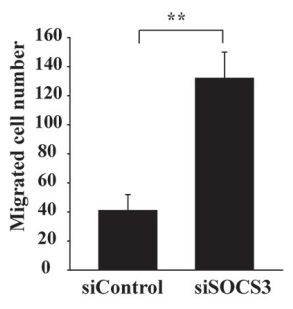

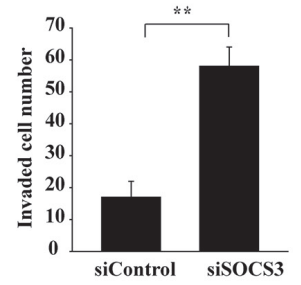

F

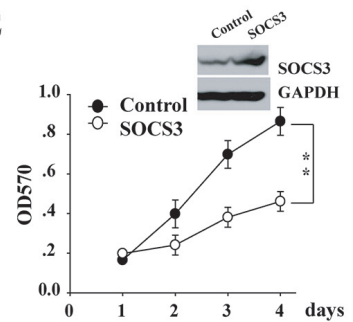

H

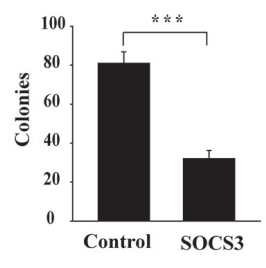

G

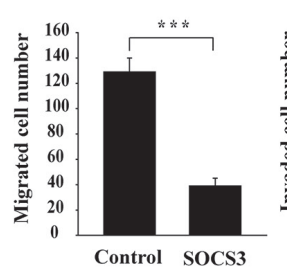

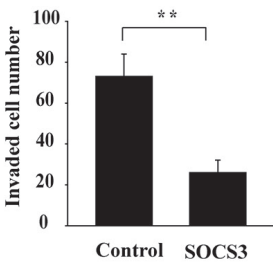

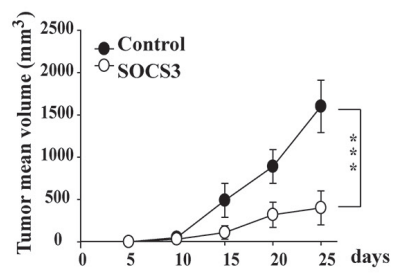

Control SOCS3

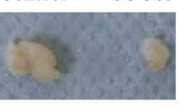

Figure 3: miR-455-5p promotes NSCLC tumorigenesis via targeting SOCS3. (A-D) RNAi knockdown of SOCS3 in H460 cells promoted the cell proliferation (A), soft-agar colony formation assays (B), Transwell cell migration and invasion (C), and xenograft tumor growth in nude mice (D) (E-H), ectopic expression of SOCS3 in H1299 cells decreased the cell proliferation (E), soft-agar colony formation assays $(\mathrm{F})$, Transwell cell migration and invasion $(\mathrm{G})$, and xenograft tumor growth in nude mice $(\mathrm{H})$. Error bars represent $\mathrm{SD} .{ }^{* *}$, $\mathrm{P}<0.01 ;{ }^{* * *}, \mathrm{P}<0.001$. Results shown are representative of three independent experiments. 
5 pmimic transfection but no change in control cells (Figure 2C). However, inhibition of miR-455-5p by antimiR-455-5p in H1299 cells, an increased endogenous miR-455-5p expression, resulted in enhanced SOCS3 expression (3 fold) (Figure 2D). These results show that SOCS3 is a direct target of miR-455-5p.

\section{miR-455-5p promotes tumorigenesis by targeting SOCS3 in NSCLC}

As a previous study showed that miR-455-5p inhibits the tumor suppressor gene CPEB1 expression to facilitate melanoma metastasis [16], we next test whether the targeting of SOCS3 by miR-455-5p is important for NSCLC. We found that SOCS3 knockdown in H460 cells with higher level of SOCS3, significantly promoted cell proliferation (Figure 3A, Supplementary Figure 2), anchorage-independent growth (3 fold; Figure 3B), cell migration and invasion (Figure 3C), and tumor growth in vivo (Figure 3D), suggesting a tumor-suppressive role of SOCS3 in NSCLC cells. Additionally, overexpression of Flag-SOCS3 in H1299 cells with lower SOCS3 level, dramatically reduced cell proliferation, colony formation, cell migration and invasion, and tumor growth in vivo (Figure 3E-3H). Thus, SOCS3 plays a tumor-suppressive function in NSCLC cells.

Next, we determined whether downregulation of SOCS3 by miR-455-5p is also functional for NSCLC cells. Overexpression of miR-455-5p in H460 cells promoted cell proliferation, colony formation, cell migration and invasion, and tumor growth in vivo (Figure 4A-4D). Moreover, the pro-tumorigenic effect of miR-455-5p was inhibited when co-transfection of a miR-455-5p-resistant form of SOCS3 (Figure 4A-4D). These results suggest that targeting SOCS3 is helpful for pro-tumorigenic function of miR-455-5p.

\section{SOCS3 is significantly down-regulated and is correlated to poor clinical outcomes}

IHC assay showed that nuclear SOCS3 was strongly expressed in most non-cancerous lung tissues, however, SOCS3 was less found in NSCLC tissues (Figure 5A and 5B). NSCLC patients with high SOCS3 levels showed
A



B



C
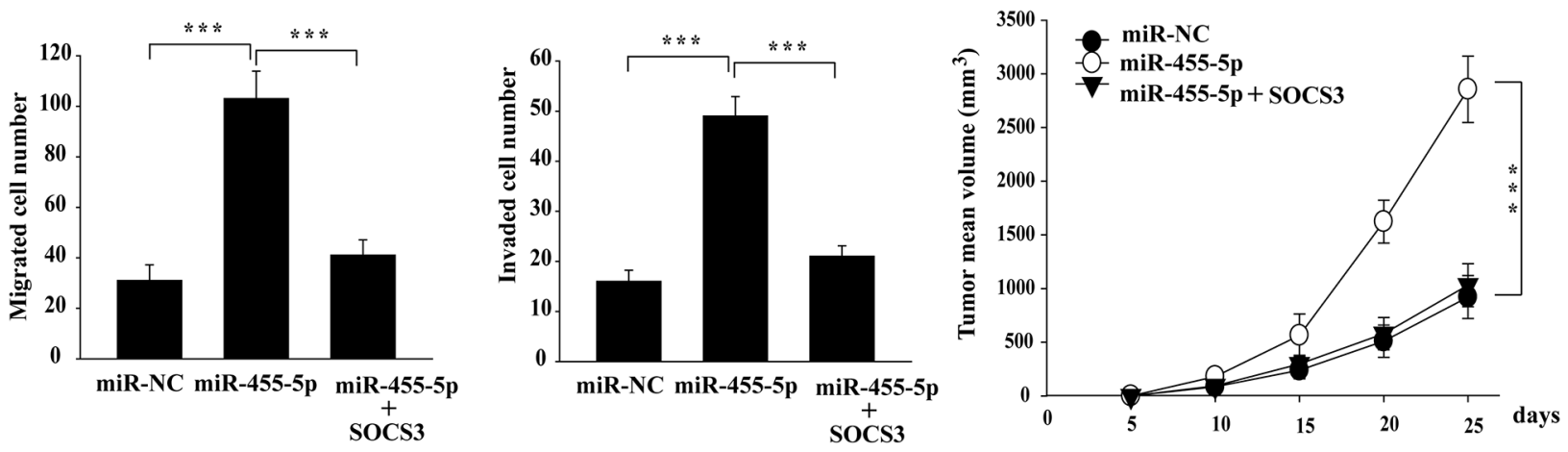

Figure 4: The miR-455-5p-SOCS3 axis is of functional importance in regulating tumorigenesis in NSCLC cells. (A-D) ectopic expression of SOCS3 in miR-455-5p mimics-transfected H460 cells significantly attenuated the effect of miR-455-5p on the cell proliferation (A), soft-agar colony formation assays (B), Transwell cell migration and invasion (C), and xenograft tumor growth in nude mice (D). Error bars represent SD. ${ }^{* *}, \mathrm{P}<0.01{ }^{* * *}, \mathrm{P}<0.001$. Results shown are representative of three independent experiments. 
much longer median overall survival, compared with those with low SOCS3 levels ( $P<0.05$, Figure 5C).

\section{Inverse correlation between miR-455-5p and SOCS3 expression in NSCLC patients}

By Pearson correlation analysis, we further found there is a significant negative correlation between miR-455$5 \mathrm{p}$ and SOCS3 mRNA levels in tumor tissues (Figure 6A). Consistently, high miR-455-5p levels in NSCLC tumors displayed weak SOCS3 IHC staining (Figure 6B, left), however, low miR-455-5p expression displayed strong SOCS3 staining (Figure 6B, right). These results suggest that there is an inverse correlation in miR-455-5p and SOCS3.

\section{ERK signaling pathway promotes miR-455-5p expression}

Next, we tested whether ERK signaling regulates miR-455-5 expression. Phorbol 12-myristate 13-acetate (PMA) in H460 cells was used to activate ERK1/2. The expression of miR-455-5p was significantly up-regulated
(7 fold) with PMA stimulated compared to the control (Figure 7A). PMA treatment increased pERK and c-Jun expressions at protein level (Figure 7A). Additionally, a luciferase reporter including miR-455-5p promoter region significantly up-regulated luciferase activity (5 fold) compared to the control vector, and the effect was much stronger with PMA treatment (3 fold; Figure 7B), suggesting the ERK signaling pathway might regulate miR-455-5p. To explore whether c-Jun plays a role in the transcription of miR-455-5p, we found that knockdown of c-Jun notably inhibited miR-455-5p promoter vector-induced luciferase activity (3 folds; Figure 7C). Furthermore, knockdown of c-Jun decreased miR-455-5p expression in H460 cells (3.3 fold; Figure 7D). These results suggest that ERK signaling pathway promotes miR-455-5p expression.

\section{DISCUSSION}

Lung cancer is very common lethal types of cancer [20-22]. It is very valuable to further explore the potential mechanisms of NSCLC due to its poor clinical outcome [23-25]. Recently, many reports have indicated

A

Adjacent lung tissue

Lung cancer

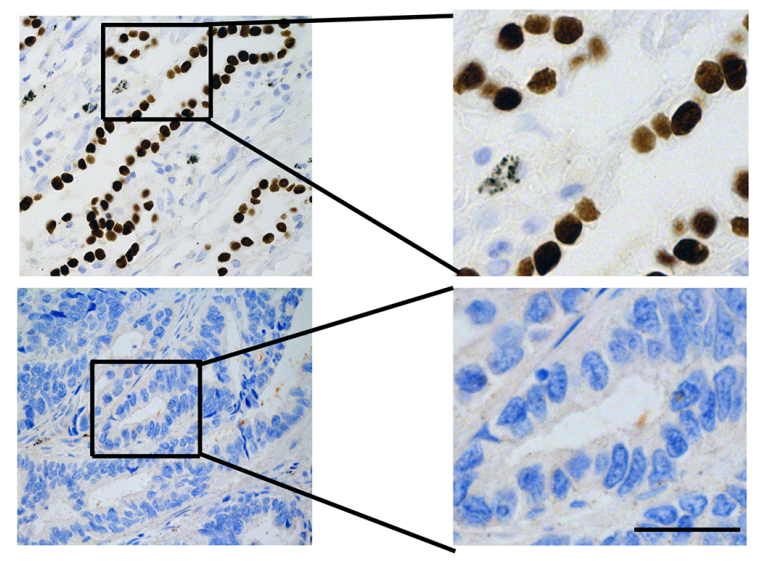

B

C
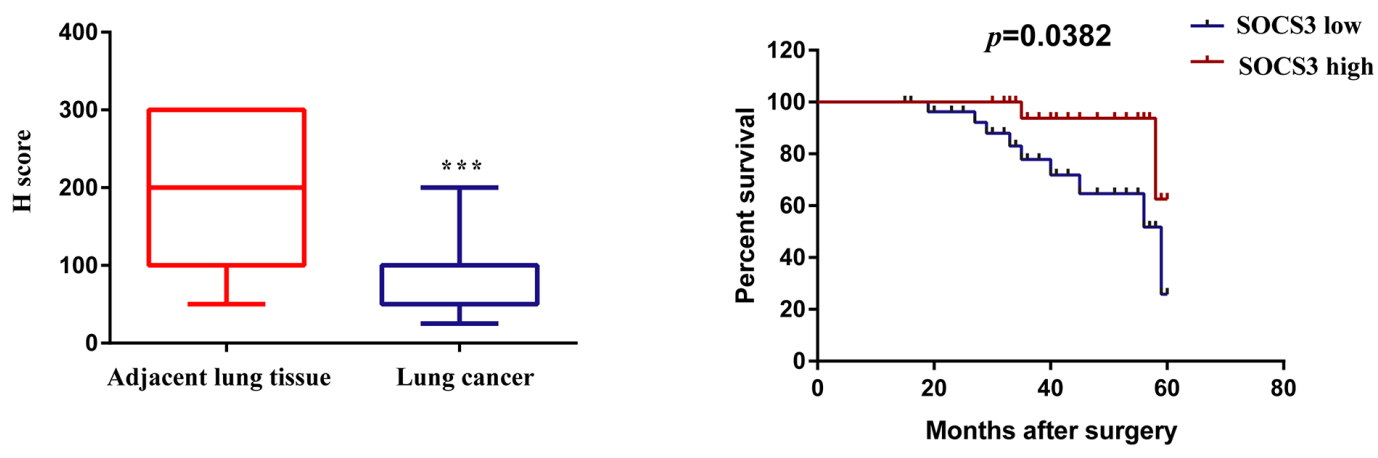

Figure 5: SOCS3 is significantly down-regulated in NSCLC tissues and is correlated with poor clinical outcomes. (A) Representative immunostaining of SOCS3 expression in human NSCLC tissues and corresponding adjacent lung tissues. Bar=200 $\mu \mathrm{m}$. (B) Quantitative analysis of SOCS3 expression in 57 cases of paired NSCLC tissues and their corresponding adjacent lung tissues. The P-value corresponds to the comparison of SOCS3 expression between the NSCLC tissues and corresponding adjacent lung tissues. ${ }^{* * *}, \mathrm{P}<0.001$. (C) Kaplan-Meier analysis of overall survival in all NSCLC patients according to SOCS3 protein level. 
A

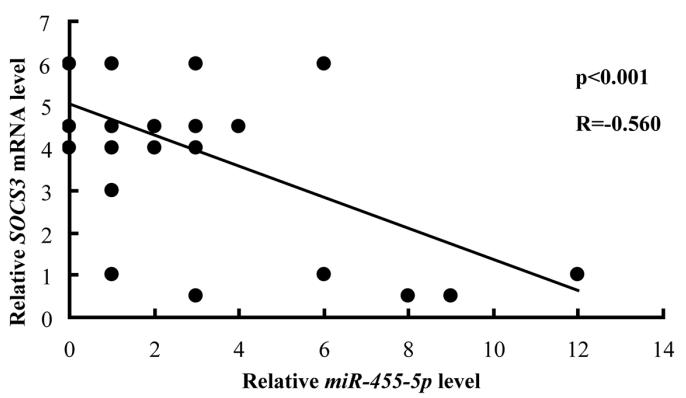

B


Figure 6: Negative correlation between miR-455-5p and SOCS3 expression in NSCLC patients. (A) miR-455-5p and SOCS3 mRNA levels were inversely correlated in NSCLC tumors. The qRT-PCR were analyzed and shown as relative miR-455-5p levels of the $\mathrm{Ct}$ (cycle threshold) values, which were then converted as fold change. (B) representative images of SOCS3 immunohistochemical

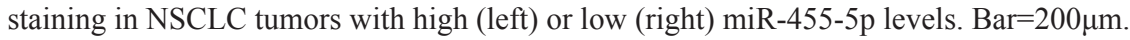

A

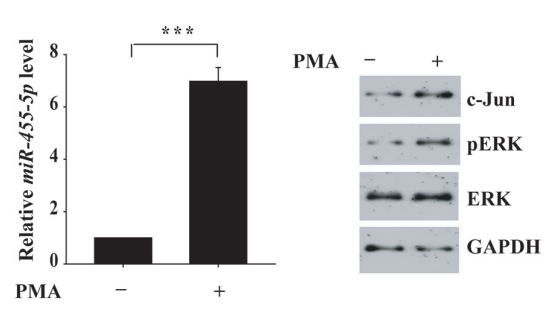

C

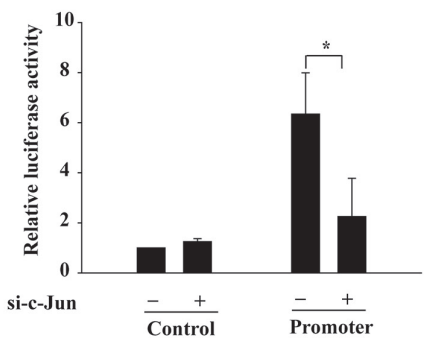

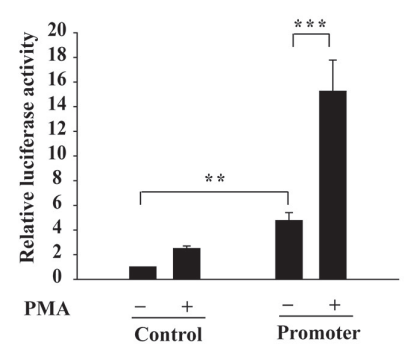

D



Figure 7: Activation of ERK signaling pathway induces miR-455-5p expression. (A) quantitative RT-PCR for H460 cells with and without PMA treatment to measure miR-455-5p expression. ${ }^{* * *}, \mathrm{P}<0.001$. (B) Luciferase reporter construct-containing promoter region of miR-455-5p and control vector were transfected into $293 \mathrm{~T}$ cells and treated with PMA for $24 \mathrm{~h} .{ }^{* *}, \mathrm{P}<0.01 ;{ }^{* * *}, \mathrm{P}<0.001$. (C) Luciferase reporter constructs containing miR-455-5p promoter and control vector were cotransfected with c-Jun siRNA into the $293 \mathrm{~T}$ cells. ${ }^{*}, \mathrm{P}<0.05$. (D) quantitative RT-PCR for H460 cells with and without c-Jun knockdown to measure miR-455-5p expression. ${ }^{* * *}, \mathrm{P}<0.001$. 
that many miRNAs play key roles in tumor progression, including lung cancer [26-36].

In this study, we demonstrated that the miR-455$5 p$ expression is significantly up-regulated in NSCLC patient samples. Serval reports have showed that miR$455-5 p$ is abnormal expression in cancers. For example, Sand et al. showed that miR-455-5p was significantly increased in basal cell carcinoma (BCC) of the skin [13]. Shoshan et al. reported that miR-455 overexpression leads to increased melanoma tumor growth and metastasis [16]. Boisen et al. found that lower miR-455$5 p$ expression was predictive of improved outcomes in metastatic colorectal cancer [37]. We found that miR-455-5p is an oncogene in lung caner. miR-455-5p significantly promoted tumor growth in the subcutaneous xenograft model. Interestingly, Li et al found that miR455 was dramatically down-regulated by using NSCLC cell lines and tissues. Furthermore, miR-455 suppressed the proliferation, migration, and invasion through targeting ZEB1 in NSCLC [38]. Because a miRNA precursor generally induces two major miRNA groups, miRNA-5p and miRNA-3p, we speculated that miR$455-5 p$ is a tumor promoter and miR-455-3p is a tumor suppressor. Indeed, miR-455-3p has been reported as a tumor suppressor $[39,40]$. Taken together, our results suggest that miR-455-5p functioned as an oncogene in NSCLC.

The JAK-STAT pathway plays critical roles in the initiation and development of tumors. The SOCS3 protein is an inhibitor of activation of the JAK-STAT pathway [41, 42]. SOCS3 inhibited tumor cell proliferation, migration, and invasion in many tumor types [17-19]. We further found c-Jun binds to the miR-455-5p promoter region. Luciferase reporter assays showed that ERK activation markedly promoted the luciferase activity of a miR-455-5p promoter reporter. Accordingly, our in vitro analyses demonstrated increased miR-455-5p expression after activation of ERK in lung cancer cells. Overall, our results suggest that upregulated expression of miR-455-5p might be attributable to the binding of ERK to the miR-455-5p promoter.

Overall, miR-455-5p promotes the growth, migration, invasion through directly targeting SOCS3. We further show that ERK signaling controls the regulation of miR-455-5p expression in NSCLC. Hence, the ERK/ miR-455-5p/SOCS3 pathway might be an ideal target for therapeutic intervention in certain NSCLC patients.

\section{MATERIALS AND METHODS}

\section{Ethics statement}

Patient information and samples were obtained with written informed consent. Each patient in this study gave written informed consent to publish these case details. The research was approved by the ethics committee of Harbin Medical University Cancer Hospital.

\section{Clinical NSCLC tissue samples}

Lung cancer specimens and matched adjacent noncancerous tissues $(\mathrm{n}=79)$ were collected from patients with NSCLC in Harbin Medical University Cancer Hospital from 2009 to 2012 . The tissues were stored at $-80^{\circ} \mathrm{C}$ until use. All samples were from patients who had not undergone preoperative radiotherapy or chemotherapy. The pathological staging of the 79 tumors was performed according to the tumor-node-metastasis (TNM) staging system.

\section{Cell lines}

Human NSCLC cell lines, including H460, SKMES-1, SPCA-1, A549, and H1299, the normal lung cell line 16HBE and the human embryonic kidney cell line 293T were purchased from American Type Culture Collection (ATCC) and maintained in DMEM supplemented with $10 \%$ fetal bovine serum (FBS) (Invitrogen) containing 100 units $/ \mathrm{ml}$ penicillin and 100 units $/ \mathrm{ml}$ streptomycin (Sigma) at $37^{\circ} \mathrm{C}$ with $5 \% \mathrm{CO}_{2}$.

\section{Transfection}

Hsa-miR-455-5p mimics and a hsa-miR-455-5p inhibitor were purchased from Sigma-Aldrich. SOCS3 siRNA was obtained from Life Technologies. H460 and H1299 cells were transfected using X-tremeGENE (Roche Applied Science), and HEK293T cells were transfected using Lipofectamine 2000 (Invitrogen) according to the manufacturer's directions. SOCS3 siRNA was transfected into cells for 24 hours using Lipofectamine RNAiMAX Reagent (Invitrogen).

\section{RNA preparation and quantitative real-time PCR}

Total RNAs were obtained using the TRIzol reagent (Invitrogen). Reverse transcription of RNA was performed using the ImProm-II reverse transcription system (Promega) according to the manufacturer's instructions.

Quantitative real-time PCR was used to precisely quantify miR-455-5p expression in human NSCLC tissues or cell lines. Quantitative RT-PCR was performed with SYBR Green reagents (Takara, Japan) in a 7500 real-time PCR system from Applied Biosystems. The $2^{-\Delta \Delta C T}$ method was used to measure the miR-455-5p gene expression compared with the endogenous controls (U6 non-coding small nuclear RNA). All primers for miR-455-5p and the U6 genes were designed by Primer Premier 5.0 and synthesized by Shanghai GenePharma.

\section{Protein extraction and western blot analysis}

Cellular proteins were extracted from cultured cells with lysis buffer (50 mM Tris, $\mathrm{pH} 8.0,150 \mathrm{mM} \mathrm{NaCl}$, $5 \mathrm{mM}$ EDTA, $50 \mathrm{mM} \mathrm{NaF}$, and $0.1 \% \mathrm{NP}-40$ ). Protein 
samples were separated in sodium dodecyl sulfate (SDS)PAGE and transferred to nitrocellulose filter membranes (Millipore, USA). After blocking in phosphate buffered saline (PBS)/Tween-20 containing 5\% nonfat milk, the membranes were incubated with the following primary antibodies: SOCO3 (Santa Cruz Biotech), phosphorERK1/2(Cell Signaling Technology), c-Jun (Cell Signaling Technology), GAPDH (Santa Cruz Biotech). Subsequent visualization was detected with Super Signal West Femto Maximum Sensitivity Substrate (Thermo, Japan).

\section{Dual-luciferase reporter assay}

The 3'-untranslated region (UTR) WT and mutant of human SOCS3 were amplified from human genomic DNA and individually inserted into the pmiR-RB-REPORTTM (Ribobio, Guangzhou, China). For reporter assays, cells were co-transfected with wild-type (mutant) reporter plasmid and miR-RiboTM mimics (miR-RiboTM negative control) using Lipofectamine 2000 (Invitrogen). The cells were then harvested and analyzed with the Dual Luciferase Reporter Assay system (Promega). All assays were performed in triplicate, and all values were normalized for transfection efficiency against Renilla luciferase activities. Primers are provided in Supplementary Table 1.

\section{In vitro cell proliferation assays}

For cell proliferation assays, cells were seeded into each well of a 96-well plate (2000 per well) and the cell proliferation ability was determined using the Cell Counting Kit-8 (CCK8) Assay Kit (Dojindo Corp, Japan): $10 \mu \mathrm{l}$ of the kit reagent dissolved with $100 \mu \mathrm{l}$ DMEM was added to each well, and $2 \mathrm{~h}$ later the absorbance was measured at $570 \mathrm{~nm}$ to calculate the number of cells.

\section{Colony formation assay}

Cells were seeded at approximately 2000 cells/well in a 6-well plate. After 14 days incubation, the cells were washed with PBS twice, fixed with methanol for $10 \mathrm{~min}$, and stained with $0.5 \%$ crystal violet for $20 \mathrm{~min}$ at room temperature. The visible colonies were counted.

\section{In vitro transwell migration and invasion assays}

Cell migration and invasion assays were performed using a 24-well plate with 8 - $\mu \mathrm{m}$ pore size chamber inserts (Corning). For migration assays, $5 \times 10^{4}$ cells were placed into the upper chamber per well with the non-coated membrane. For invasion assays, $1 \times 10^{5}$ cells were placed into the upper chamber per well with a Matrigel-coated membrane that was diluted with serum-free culture medium. In both assays, cells were suspended in $200 \mu \mathrm{l}$ of DMEM without FBS when they were seeded into the upper chamber. In the lower chamber, $800 \mu$ of DMEM supplemented with $10 \%$ FBS was added. After incubation for $16 \mathrm{~h}$ at $37^{\circ} \mathrm{C}$ and $5 \% \mathrm{CO}_{2}$, the membrane inserts were removed from the plate, and non-migrated or noninvading cells were removed from the upper surface of the membrane. Cells that moved to the bottom surface of the chamber were fixed with $100 \%$ methanol for $20 \mathrm{~min}$ and stained with $0.1 \%$ crystal violet for $30 \mathrm{~min}$. Then, the cells were imaged and counted in at least 8 random fields. The assays were conducted three independent times.

\section{Immunohistochemistry}

Immunohistochemistry of patient tissue sections was performed as recently described [20]. The dewaxed 5 - $\mu \mathrm{m}$ sections were subjected to an antigen-demasking procedure of brief, high temperature heating of the sections immersed in citrate buffer. Endogenous peroxidases were blocked with $0.3 \%$ hydrogen peroxide, and nonspecific binding was blocked with 5\% normal goat serum and $2 \%$ BSA in phosphate-buffered saline (PBS). Sections were then incubated for 2 hours at room temperature with anti-SOCO3 antibody (1:50; Santa Cruz Biotech). After washing with PBS, sections were incubated with biotinylated secondary antibody, followed by a further incubation with the streptavidin-horseradish peroxidase complex. The sections were then immersed in DAB for 5 to 10 minutes, counterstained with 10\% Mayer hematoxylin, dehydrated, and mounted in crystal mount.

\section{Xenograft assays in nude mice}

All animal work was performed in accordance with the institutional ethical guidelines for animal experimentation. Twenty-four hours after transfection of RNA oligonucleotide and/or plasmid DNA, approximately $2 \times 10^{6}$ cells were suspended in $100 \mathrm{~mL}$ of DMEM and then injected subcutaneously into male BALB/c athymic nude mice at 6 to 8 weeks of age. Six mice were included in each experimental group. Tumor growth rates were examined every 5 days for 25 days. Tumor growth rates were analyzed by measuring tumor length (L) and width (W) and calculating the volume with the formula $L W^{2} / 2$.

\section{Statistical analysis}

All results were presented as the mean \pm standard error of the mean (SEM). A Student $t$ test was performed to compare the differences between treated groups relative to their paired controls. One-way ANOVA was used to analyze tumor growth data. $P$ values are indicated in the text and figures above the two groups compared with a value $<0.05$ (denoted by asterisks) considered significant.

\section{CONFLICTS OF INTEREST}

The authors declare that they have no conflicts of interest. 


\section{FUNDING}

This work was supported by National Natural Science Foundation of China (81172215 and 81172818).

\section{REFERENCES}

1. Torre LA, Bray F, Siegel RL, Ferlay J, Lortet-Tieulent J, Jemal A. Global cancer statistics, 2012. CA Cancer J Clin. 2015; 65:87-108.

2. Siegel RL, Miller KD, Jemal A. Cancer statistics, 2015. CA Cancer J Clin. 2015; 65:5-29.

3. Molina JR, Yang P, Cassivi SD, Schild SE, Adjei AA. Nonsmall cell lung cancer: epidemiology, risk factors, treatment, and survivorship. Mayo Clin Proc. 2008; 83:584-594.

4. Gupta GP, Massague J. Cancer metastasis: building a framework. Cell. 2006; 127:679-695.

5. Hsiao SH, Chung CL, Chou YT, Lee HL, Lin SE, Liu HE. Identification of subgroup patients with stage IIIB/IV nonsmall cell lung cancer at higher risk for brain metastases. Lung Cancer. 2013; 82:319-323.

6. Wu L, Belasco JG. Let me count the ways: mechanisms of gene regulation by miRNAs and siRNAs. Mol Cell. 2008; 29:1-7.

7. Bartel DP. MicroRNAs: genomics, biogenesis, mechanism, and function. Cell. 2004; 116:281-297.

8. Lewis BP, Burge CB, Bartel DP. Conserved seedpairing, often flanked by adenosines, indicates thatthousands of human genes are microRNA targets. Cell. 2005; 120:15-20.

9. Garzon R, Marcucci G, Croce CM. Targeting microRNAs in cancer: rationale, strategies and challenges. Nat Rev Drug Discov. 2010; 9:775-789.

10. Kasinski AL, Slack FJ. Epigenetics and genetics. MicroRNAs en route to the clinic: progress in validating and targeting microRNAs for cancer therapy. Nat Rev Cancer. 2011; 11:849-864.

11. Croce CM. Causes and consequences of microRNA dysregulation in cancer. Nat Rev Genet. 2009; 10:704-714.

12. Hiroki E, Akahira J, Suzuki F, Nagase S, Ito K, Suzuki T, Sasano H, Yaegashi N. Changes in microRNA expression levels correlate with clinicopathological features and prognoses in endometrial serous adenocarcinomas. Cancer Sci. 2010; 101:241-249.

13. Sand M, Skrygan M, Sand D, Georgas D, Hahn SA, Gambichler T, Altmeyer P, Bechara FG. Expression of microRNAs in basal cell carcinoma. Br J Dermatol. 2012; 167:847-855.

14. Saito K, Inagaki K, Kamimoto T, Ito Y, Sugita T, Nakajo S, Hirasawa A, Iwamaru A, Ishikura T, Hanaoka H, Okubo K, Onozaki T, Zama T. MicroRNA-196a is a putative diagnostic biomarker and therapeutic target for laryngeal cancer. PLoS One. 2013; 8:e71480.
15. Chiu LY, Kishnani PS, Chuang TP, Tang CY, Liu CY, Bali D, Koeberl D, Austin S, Boyette K, Weinstein DA, Murphy E, Yao A, Chen YT, et al. Identification of differentially expressed microRNAs in human hepatocellular adenoma associated with type I glycogen storage disease: a potential utility as biomarkers. J Gastroenterol. 2014; 49:1274-1284.

16. Shoshan E, Mobley AK, Braeuer RR, Kamiya T, Huang L, Vasquez ME, Salameh A, Lee HJ, Kim SJ, Ivan C, Velazquez-Torres G, Nip KM, Zhu K, et al. Reduced adenosine-to-inosine miR-455-5p editing promotes melanoma growth and metastasis. Nat Cell Biol. 2015; 17:311-321.

17. Niwa Y, Kanda H, Shikauchi Y, Saiura A, Matsubara K, Kitagawa T, Yamamoto J, Kubo T, Yoshikawa H. Methylation silencing of SOCS-3 promotes cell growth and migration by enhancing JAK/STAT and FAK signalings in human hepatocellular carcinoma. Oncogene. 2005; 24:6406-6417.

18. Zhang WN, Wang L, Wang Q, Luo X, Fang DF, Chen Y, Pan X, Man JH, Xia Q, Jin BF, Li WH, Li T, Liang B, et al. CUEDC2 (CUE domain-containing 2) and SOCS3 (suppressors of cytokine signaling 3) cooperate to negatively regulate Janus kinase 1 /signal transducers and activators of transcription 3 signaling. J Biol Chem. 2012; 287:382-392.

19. Kneitz B, Krebs M, Kalogirou C, Schubert M, Joniau S, van Poppel H, Lerut E, Kneitz S, Scholz CJ, Ströbel P, Gessler M, Riedmiller H, Spahn M. Survival in patients with high-risk prostate cancer is predicted by miR-221, which regulates proliferation, apoptosis, and invasion of prostate cancer cells by inhibiting IRF2 and SOCS3. Cancer Res. 2014; 74:2591-2603.

20. Nie W, Tao X, Wei H, Chen WS, Li B. The BIM deletion polymorphism is a prognostic biomarker of EGFR-TKIs response in NSCLC: a systematic review and meta-analysis. Oncotarget. 2015; 6:25696-25700. https://doi.org/10.18632/ oncotarget.4678.

21. Anagnostou VK, Brahmer JR. Cancer immunotherapy: a future paradigm shift in the treatment of non-small cell lung cancer. Clin Cancer Res. 2015; 21:976-984.

22. Stinchcombe TE, Johnson GL. MEK inhibition in non-small cell lung cancer. Lung Cancer. 2014; 86:121-125.

23. Formisano L, D'Amato V, Servetto A, Brillante S, Raimondo L, Di Mauro C, Marciano R, Orsini RC, Cosconati S, Randazzo A, Parsons SJ, Montuori N, Veneziani BM, et al. Src inhibitors act through different mechanisms in Non-Small Cell Lung Cancer models depending on EGFR and RAS mutational status. Oncotarget. 2015; 6:26090-26103. https://doi.org/10.18632/ oncotarget.4636.

24. Cui R, Kim T, Fassan M, Meng W, Sun HL, Jeon YJ, Vicentini C, Tili E, Peng Y, Scarpa A, Liang G, Zhang YK, Chakravarti A, Croce CM. MicroRNA-224 is implicated in lung cancer pathogenesis through targeting caspase- 3 and 
caspase-7. Oncotarget. 2015; 6:21802-21815. https://doi. org/10.18632/oncotarget.5224.

25. Gridelli C, Peters S, Sgambato A, Casaluce F, Adjei AA, Ciardiello F. ALK inhibitors in the treatment of advanced NSCLC. Cancer Treat Rev. 2014; 40:300-306.

26. Sun C, Huang C, Li S, Yang C, Xi Y, Wang L, Zhang F, Fu Y, Li D. Hsa-miR-326 targets CCND1 and inhibits nonsmall cell lung cancer development. Oncotarget. 2016; 7:8341-8359. https://doi.org/10.18632/oncotarget.7071.

27. Sun CC, Li SJ, Zhang F, Zhang YD, Zuo ZY, Xi YY, Wang L, Li DJ. The novel miR-9600 suppresses tumor progression and promotes paclitaxel sensitivity in nonsmall-cell lung cancer through altering STAT3 expression. Mol Ther Nucleic Acids. 2016; 5:e387.

28. Sun C, Sang M, Li S, Sun X, Yang C, Xi Y, Wang L, Zhang F, Bi Y, Fu Y, Li D. Hsa-miR-139-5p inhibits proliferation and causes apoptosis associated with down-regulation of c-Met. Oncotarget. 2015; 6:39756-39792. https://doi. org/10.18632/oncotarget.5476.

29. Sun CC, Li SJ, Yuan ZP, Li DJ. MicroRNA-346 facilitates cell growth and metastasis, and suppresses cell apoptosis in human non-small cell lung cancer by regulation of XPC/ ERK/Snail/E-cadherin pathway. Aging (Albany NY). 2016; 8:2509-2524. https://doi.org/10.18632/aging.101080.

30. Xi Y, Wang L, Sun C, Yang C, Zhang F, Li D. The novel miR-9501 inhibits cell proliferation, migration and activates apoptosis in non-small cell lung cancer. Med Oncol. 2016; 33:124.

31. Sun C, Li S, Zhang F, Xi Y, Wang L, Bi Y, Li D. Long non-coding RNA NEAT1 promotes non-small cell lung cancer progression through regulation of miR-377-3pE2F3 pathway. Oncotarget. 2016; 7:51784-51814. https:// doi.org/ 10.18632/oncotarget.10108.

32. Huang J, Sun C, Wang S, He Q, Li D. microRNA miR-10b inhibition reduces cell proliferation and promotes apoptosis in non-small cell lung cancer (NSCLC) cells. Mol Biosyst. 2015; 11:2051-2059.

33. Sun CC, Li SJ, Li DJ. Hsa-miR-134 suppresses non-small cell lung cancer (NSCLC) development through downregulation of CCND1. Oncotarget. 2016; 7:35960-35978. https://doi.org/10.18632/oncotarget.8482.
34. Sun C, Li S, Yang C, Xi Y, Wang L, Zhang F, Li D. MicroRNA-187-3p mitigates non-small cell lung cancer (NSCLC) development through down-regulation of BCL6. Biochem Biophys Res Commun. 2016; 471:82-88.

35. Sun CC, Li SJ, Zhang F, Pan JY, Wang L, Yang CL, Xi YY, Li de J. Hsa-miR-329 exerts tumor suppressor function through down-regulation of MET in non-small cell lung cancer. Oncotarget. 2016; 7:21510-21516. https://doi. org/10.18632/oncotarget.7517.

36. Sun C, Liu Z, Li S, Yang C, Xue R, Xi Y, Wang L, Wang S, He Q, Huang J, Xie S, Jiang W, Li D. Down-regulation of c-Met and Bcl2 by microRNA-206, activates apoptosis, and inhibits tumor cell proliferation, migration and colony formation. Oncotarget. 2015; 6:25533-25574. https://doi. org/10.18632/oncotarget.4575.

37. Boisen MK, Dehlendorff C, Linnemann D, Nielsen BS, Larsen JS, Osterlind K, Nielsen SE, Tarpgaard LS, Qvortrup C, Pfeiffer P, Holländer NH, Keldsen N, Hansen TF, et al. Tissue microRNAs as predictors of outcome in patients with metastatic colorectal cancer treated with first line Capecitabine and Oxaliplatin with or without Bevacizumab. PLoS One. 2014; 9:e109430.

38. Li YJ, Ping C, Tang J, Zhang W. MicroRNA-455 suppresses non-small cell lung cancer through targeting ZEB1. Cell Biol Int. 2016; 40:621-628.

39. Zhao Y, Yan M, Yun Y, Zhang J, Zhang R, Li Y, Wu X, Liu Q, Miao W, Jiang H. MicroRNA-455-3p functions as a tumor suppressor by targeting eIF4E in prostate cancer. Oncol Rep. 2017; 37:2449-2458.

40. Zheng J, Lin Z, Zhang L, Chen H. MicroRNA-455-3p inhibits tumor cell proliferation and induces apoptosis in HCT116 human colon cancer cells. Med Sci Monit. 2016; 22:4431-4437.

41. Inagaki-Ohara K, Kondo T, Ito M, Yoshimura A. SOCS, inflammation, and cancer. JAKSTAT. 2013; 2:e24053.

42. Li Y, de Haar C, Peppelenbosch MP, van der Woude CJ. SOCS3 in immune regulation of inflammatory bowel disease and inflammatory bowel disease-related cancer. Cytokine Growth Factor Rev. 2012; 23:127-138. 